\title{
Ocular fluorophotophotometry
}

Recently there have been considerable developments in the techniques and potential uses of ocular fluorophotometry for the study of endogenous ocular fluorescence (the naturally autofluorescent compounds found in the retina, lens, or cornea) or the physiological barriers that protect the eye from its external environment. Corneal epithelial or endothelial cell function can be measured by topical application of fluorescein, a technique that has been used extensively in the contact lens and pharmaceutical industry, and this method can be adapted to measure the production of tears or aqueous humour and the function of the posterior capsule barrier. Measurement of the blood-aqueous barrier (BAB) or bloodretinal barrier (BRB) requires systemic administration of fluorescein either orally or intravenously.

Much of the stimulus to the study of the BRB by vitreous fluorophotometry came from the claim in the 1970s that a preretinopathy stage of diabetic retinopathy could be found when there was excessive retinal permeability to fluorescein in the absence of overt retinopathy. Further studies, however, failed to substantiate this claim, and to some extent vitreous fluorophotometry then fell into disrepute. Many of the initial problems arose from variability in equipment, techniques, and data processing, and the resolution of these problems has stimulated more interest. The development of the Fluorotron Master by Coherent Radiation has produced a fluorophotometer that is highly accurate, reliable, gives reproducible results, and can measure fluorescence in any part of the eye. This machine has stood the test of time and has been accepted as the 'gold standard'. More recently an EEC working party on ocular fluorophotometry has standardised protocols for each ocular barrier, providing European standards and common software. It will eventually provide a large pool of normal data, so that clinical studies will be directly comparable.

The clinical role of vitreous fluorophotometry has been reestablished by work done in Chicago, Copenhagen, and at the Hammersmith Hospital in London. A single examination requires an hour with the patient, expensive equipment, and a skilled operator as well as an intravenous injection and at least two blood specimens, so its role is limited to clinical research. Vitreous fluorophotometry reflects permeability changes in the central $30^{\circ}$ of the fundus and can be performed only in the presence of an intact vitreous body, because a posterior vitreous detachment produces pooling and mixing artefacts which defy analysis. This is not, however, a tremendous practical problem, as a posterior vitreous detachment is easily identified by the fluorescent pattern in the gel, and these eyes excluded.

The Hammersmith group has shown that the degree of retinal permeability correlates with the severity of background diabetic retinopathy, and, particularly in the macular area, it provides a useful objective index of the extent of diabetic background retinopathy. ${ }^{2}$ It thus confirms earlier results from Chicago which showed a good correlation between BRB permeability, visual acuity, and the extent of retinal thickening in diabetic macular oedema. ${ }^{3}$ This has become particularly relevant with the advent of clinical trials of various aldose reductase inhibitors in diabetic retinopathy. Studies in Copenhagen have shown that antihypertensive treatment lowers increased BRB permeability in diabetic eyes and that lowering of the blood pressure within the normal range with captopril tends to stabilise the BRB in diabetic patients, whereas the BRB of untreated patients tends to deteriorate. ${ }^{+}$

These observations provide an insight into how the deleterious effects of systemic hypertension are mediated in diabetic retinopathy. Abnormal permeability of the BRB can be found in a wide variety of ocular diseases, but its measurement is not worthwhile unless it is part of the primary disease process or provides a means to study disease progression or treatment. It does, for instance, provide an excellent method of quantifying recovery of the BRB after central retinal vein occlusion. ${ }^{5}$ Quantification of damage to the blood-aqueous barrier has wide implications in anterior segment surgery and inflammatory eye disease. The BAB is much more permeable than the $\mathrm{BRB}$, and different problems are encountered in its measurement. In normal eyes the blood vessels contributing to the barrier appear to be about 10 times more permeable to fluorescein than those of the BRB. ${ }^{6}$ Intravenous fluorescein is rapidly bound to albumin in the plasma and also metabolised to fluorescein glucuronide, so that one hour later only about $17 \%$ of the dose is still present as free fluorescein. These metabolites have less fluorescence than free fluorescein (30\% and 5\% respectively) and are more water soluble, so that the kinetics of their inward and outward transport across the $\mathrm{BAB}$ are different from those of free fluorescein. In the normal eye most of the fluorescence in the aqueous humour after intravenous fluorescein is due to free fluorescein, principally leaking from the iris vessels, but in the pathological eye this is no longer the case, as significant contributions can be made through the ciliary body and by the other metabolites. For this reason elegant equations to explain the $\mathrm{BAB}$ in the normal eye cannot be applied, and the $\mathrm{BAB}$ has been variously measured as a permeability or diffusion coefficient of varying complexity, the ratio of fluorescence between a normal and abnormal eye, a peak level of fluorescence, or a percentage change from a previous value. The recent EEC working party has chosen a diffusion coefficient to standardise on.

Fluorophotometry for measurement of the BAB may well, though, be soon superseded by laser devices which measure the light scattering effect of cells and protein in the anterior chamber. Kowa have produced a commercial machine which scans a small volume of the anterior chamber with a $\mathrm{He}-\mathrm{Ne}$ laser and measures reflected light from material within the anterior chamber by a sensitive photomultiplier. Mathematical analysis of the reflected photons distinguishes protein concentration from the cell count. The technique has the enormous advantages of being entirely non-invasive and extremely rapid and easy to perform, and initial work with the equipment seems to support the high expectations held for it and the expense. ${ }^{\text {. }}$

Lens autofluorescence has been a neglected area of study. There has been speculation that it might provide a long term index of diabetic control reflecting blood sugar changes over years which, if substantiated, would be of great relevance to the prediction of diabetic complications.

New developments in fluorophotometry require better instruments with increased resolution to distinguish inner and outer BRB function and differential spectroscopy 
to separate the spectrum of fluorescein from fluorescein glucuronide, which would provide another molecule with different pharmokinetics with which to study the BRB. Further developments in ocular spectroscopy have a ring of science fiction about them, but the technology is rapidly becoming available - for instance, to measure melanin and lipofuscin concentrations in the retina by time resolved spectroscopy, in which the molecule is stimulated by monochromatic light, and the time delay of the resultant fluorescence is analysed in pico or nano seconds. It is not beyond the realms of possibility to suggest it will be possible to perform in vivo immunofluorescence or fluorocytometry on the aqueous humour.

Medical Eye Unit,

St Thomas's Hospital

London SE1 7EH
1 Dalgaard P, Barker VA, Lund-Anderson H. Vitreous fluorophotometry mathematical analysis of the effect of peripheral leakage on axial scans. Invest Ophthalmol Vis Sci 1989; 30: 1522-6.

2 Plehwe WE, Sleightholm MA, Kohner EM. Does vitreous fluorophotometry reflect the severity of diabetic retinopathy? $B r F$ Ophthalmol 1989; 73: 255-60.

3 Smith RT, Lee CM, Charles HC, et al. Quantification of diabetic macular oedema. Arch Ophthalmol 1987; 105: 218-22.

4 Parving HH, Larsen M, Hommel E, Lund-Anderson H. Effect of antihypertensive treatment on blood retinal barrier permeability to fluorescein in hypertensive type I (insulin dependent) diabetic patients with background retinopathy. Diabetologica 1989; 32: 440-4.

5 Chalal PS, Fallon TJ, Kohner EM. Measurement of blood retinal barrier function in central retinal vein occlusion. Arch Ophthalmol 1986; 104: 554-7.

6 van Best JA, Lappelhof JP, Laterveer L, Ooster-hins JA. Blood aqueous barrier permeability vs age by fluorophotometry. Curr Eye Res 1987; 6: 855-63.

7 Sawa M, Tsurimaki Y, Tsuru T, Shimiza $H$. New quantitative method to determine protein concentration and cell number in the aqueous in vivo. $\mathcal{F}_{p n} \mathcal{F}$ Ophthalmol 1988; 32: 132-42.

8 Shah SM, Spalton DJ, Smith SE. Factors affecting the interpretation of results from the Kowa FC 1000 Laser Cell Flare Meter in normal eyes (In press). 